\title{
Development of Teaching Materials for Teachers about Small Astronomical Telescopes
}

\author{
Masamitsu Ohyama ${ }^{1, *}$ \\ ${ }^{1}$ Faculty of Education, Shiga University, 2-5-1 Hiratsu, Otsu, Shiga, Japan
}

\begin{abstract}
Textbooks used in elementary and junior high schools must be written based on the Courses of Study in Japan. Science textbooks show observation of the moon and the sun using a small astronomical telescope. However, most in-service teachers have never used an astronomical telescope. Teachers have to use it safely to secure the safety of pupils, students, and teachers. I examined wrong and unsafe acts while in-service teachers and students assembled astronomical telescopes. Then I developed digital teaching materials for teachers including risks caused by wrong acts.
\end{abstract}

\section{Introduction}

In Japan, the Courses of Study for elementary and junior high schools are prepared by the Ministry of Education, Culture, Sports, Science and Technology. It is the standard of curriculum in elementary and secondary education. Textbooks used in elementary and junior high schools must be written based on the Courses of Study. Teachers of elementary and junior high schools have to teach the contents of the textbooks in Japan.

The Courses of Study for elementary and junior high schools were revised in March 2008 [1-4]. Pupils begin to learn science in Grade 3, and learn content in astronomy in Grades 4 and 6. According to the Course of Study [2], they learn the following content in Grade 4:

(1) The shape of the moon appears to change day to day, and its position changes throughout the day.

(2) There are stars in the sky with different levels of brightness and color.

(3) The alignment of a cluster of stars does not change but the position of the cluster changes throughout the day.

The objectives for the content are to help pupils develop their ideas about the characteristics and movement of the moon and stars through the observation of the moon and stars, and through the exploration of the position of the moon and the color, brightness and position of stars. The science textbooks of Grade 4 show the observation of the moon and stars with the naked eye.

Pupils learn the following content in Grade 6:

(1) The sun is located on the bright side of the moon. The moon phase changes depending on the positional relationship between the moon and the sun.

\footnotetext{
* ohyama@edu.shiga-u.ac.jp
} 
(2) The condition of the moon's surface is different from that of the sun. The objectives for the content are to help pupils develop their ideas about the moon phase and conditions of the surface through the observation of the moon and the sun and through the examination of the location and the phases of the moon and the location of the sun. The science textbooks of Grade 6 show the observation of the moon with small astronomical telescopes and/or binoculars and that of the sun with solar filters.

Students learn content on astronomy in the third year of junior high school and the content is as follows:

(1) The relation between the rotation of the Earth and the diurnal motion of celestial bodies.

(2) The relation between the revolution and the axial tilt of the Earth and the annual motion of constellations and the changes in the sun's culmination height.

(3) The characteristics of the Sun based on records of student observations and other data.

(4) The relation between the moon's revolutions and the causes for the cycle phases of the moon.

(5) The characteristics of the planets and stars.

The objectives for the content are to enable students to consider the Earth's movement through observations of nearby celestial bodies, and to understand characteristics of the sun and planets. The science textbooks of the third year of junior high school show the observations of the solar surface and sunspots using solar projection through small astronomical telescopes.

Both science teachers of elementary and junior high schools need to know how to use a small astronomical telescope. However, they have never used it, and it is difficult to understand how to assemble an equatorial telescope.

At the school teachers have to use an astronomical telescope safely. Therefore, I examined wrong and unsafe acts while students and in-service teachers assembled astronomical telescopes, especially equatorial telescope. Then I developed digital teaching materials for teachers including risks by wrong acts.

\section{Examination of wrong and unsafe acts}

Most in-service teachers and students have never used small astronomical telescopes. Some teachers and students had tried to assemble small astronomical telescopes by themselves using manuals and/or books on astronomical telescopes, but some of them could not understand how to assemble them. Moreover, it is not easy for them to judge whether their acts are unsafe or not. I examined wrong and unsafe acts while students and in-service teachers assembled astronomical telescopes, especially equatorial telescopes.

\subsection{Training sessions of in-service teachers}

The observations of the moon or sun using small astronomical telescopes are recommended in science textbooks of elementary and junior high schools after the revisions of the Courses of Study in 2008. Therefore, I have held the training of in-service teachers in astronomical telescopes eleven times from 2009 to 2017 (Table 1). Some of them were requested by teachers of elementary and junior high school teachers and the board of education. Both elementary and junior high school teachers attended the training each year except for 2017. 
Table 1. The number of times of training sessions of in-service teachers of elementary and junior high school.

\begin{tabular}{|c||c|}
\hline year & $\begin{array}{c}\text { The number } \\
\text { of times }\end{array}$ \\
\hline \hline 2009 & 2 \\
\hline 2010 & 1 \\
\hline 2011 & 2 \\
\hline 2012 & 1 \\
\hline 2013 & 1 \\
\hline 2014 & 1 \\
\hline 2015 & 1 \\
\hline 2016 & 2 \\
\hline 2017 & $1^{*}$ \\
\hline
\end{tabular}

*Only teachers of elementary school attended the training in 2017.

\subsection{Classes for students}

I have given classes to teach how to use small astronomical telescopes for students of the faculty of education since 2009. Most of them became elementary and junior high school teachers. The number of the students attending each class is 25 to 35 students.

\subsection{Examination of wrong and unsafe acts}

In-service teachers and students tried to assemble small astronomical telescopes in a group of 1 to 5 people using a book on astronomical telescopes at the beginning of the training sessions and classes, respectively. It is found that they did some wrong acts while they assembled the telescopes. Moreover, even if they finished the assembly of astronomical telescopes, they were not sure if they were correct or not.

Wrong and unsafe acts while they assembled astronomical telescopes are as follows:

(1) Attachment of the equatorial mount in the wrong direction.

(2) Wrong polar alignment in the daytime.

(3) Unsafe attachment of counterweights to the counterweight shaft (Figure 1).

(4) Forgetting to tighten the thumbscrew of the counterweight shaft (Figure 2).

(5) Poor balance of the telescope with respect to the right ascension and/or declination axes. 


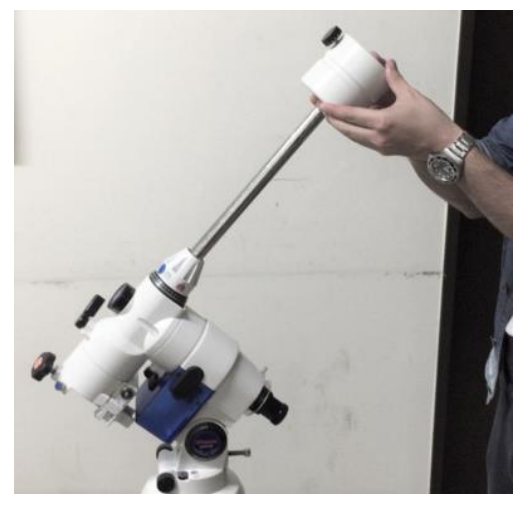

Figure 1. Unsafe attachment of counterweight

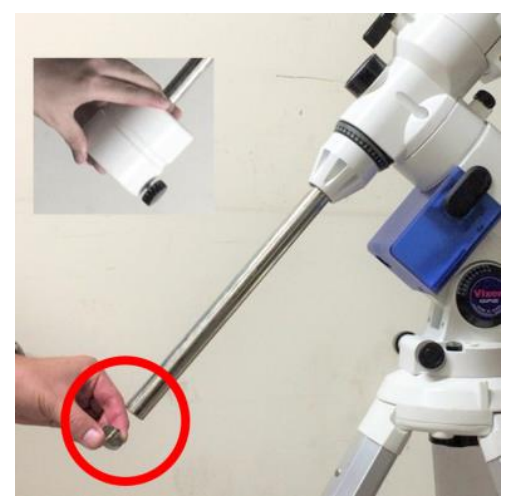

Figure 2. Thumbscrew of the counterweight shaft

When in-service teachers assemble astronomical telescopes at their schools, many pupils and students may gather around the teachers and the telescopes. The above-mentioned acts (3) and (4) are especially unsafe at the schools. In the case of (3) many teachers and students attached a counterweight to the counterweight shaft from the top. If a lock lever is loose, the counterweight would swing like a pendulum and it could hit the pupil's/student's head. In the case of (4) some teachers and students forgot to tighten the thumbscrew of the counterweight shaft. If a screw of the counter weight is loose, the counterweight could fall on a foot of a teacher, pupil, or student. Both cases cause a big accident and serious injury such as a fracture.

\section{Development of teaching materials}

Most manuals and books show only a correct way to assemble astronomical telescopes. Therefore, it is difficult for in-service teachers and students to notice risks when they do it the wrong way. Hence, I developed digital teaching materials, video materials and the related manual, on astronomical telescopes including risks by wrong acts at their schools. Table 2 shows the list of the video materials. 
Table 2. The list of the video materials.

\begin{tabular}{|c|c||l|}
\hline \multicolumn{1}{|c||}{ Section } & \multicolumn{1}{|c|}{ Title } \\
\hline \hline & 1 & $\begin{array}{l}\text { Movement of an equatorial } \\
\text { telescope }\end{array}$ \\
\hline 1 & 2 & Parts list \\
\hline 1 & 2 & $\begin{array}{l}\text { Assembly of the tripod } \\
\text { daytime }\end{array}$ \\
\hline 2 & 3 & Horizontal check \\
\hline 3 & & $\begin{array}{l}\text { Attachment of the equatorial } \\
\text { mount to the tripod }\end{array}$ \\
\hline 4 & & $\begin{array}{l}\text { Attachment of the counterweight } \\
\text { bar and counterweight }\end{array}$ \\
\hline & 1 & $\begin{array}{l}\text { Attachment of the telescope to } \\
\text { the mount }\end{array}$ \\
\hline 5 & 2 & $\begin{array}{l}\text { Attitude for the balance check } \\
\text { R.A. axis - Telescope and } \\
\text { counterweight - }\end{array}$ \\
\hline
\end{tabular}

\subsection{Video teaching materials}

The digital teaching materials were developed taking the following points into consideration:

(1) In-service teachers use astronomical telescopes at their schools.

(2) Risks by wrong acts at their schools are included to secure the safety of pupils/students and teachers.

(3) The total reproduction time of the video is within 10 minutes.

Figure 3 shows the risk of attaching a counterweight to the counterweight shaft from the top. It demonstrates the counterweight swinging like a pendulum and hitting the pupil's/student's head. 


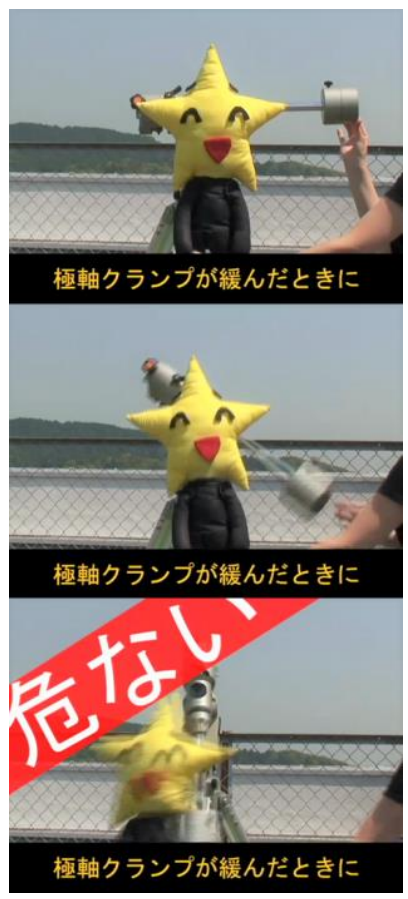

Figure 3. The risk of unsafe attachment of counterweights

Figure 4 shows the risk of forgetting to tighten the thumbscrew of the counterweight shaft. It shows a counterweight falling on a foot of a pupil/student.

I developed video teaching materials including not only the correct ways but also the risks of the wrong ways. The total reproduction time of the video is within 10 minutes. 


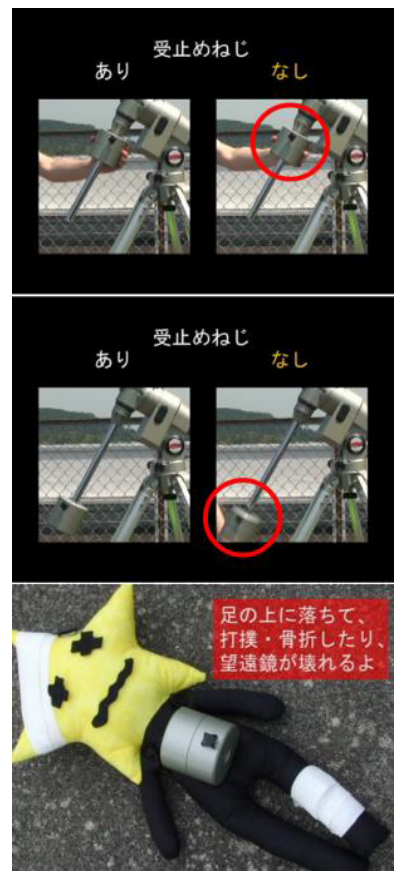

Figure 4. The risk of forgetting to tighten the thumbscrew of the counterweight shaft.

\subsection{The related manual}

I also made a digital manual related to the developed video teaching materials. Figure 5 is a part of the content which is indicated with both pictures and words at each step and which shows the page number of each step.

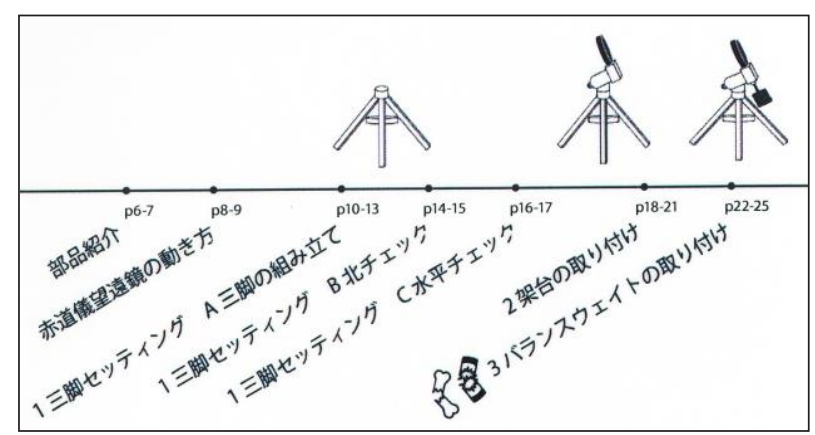

Figure 5. A part of the manual related to the developed video teaching materials.

Figure 6 shows a page of the manual which consists of several photos and short explanatory notes. The title indicating the step is written on the upper left of the page. The Arabic numeral over each photo of the upper row indicates the reproduction time of the 
developed video. The photos of the middle and lower rows indicate the enlarged photos of work points and risks by wrong acts, respectively.

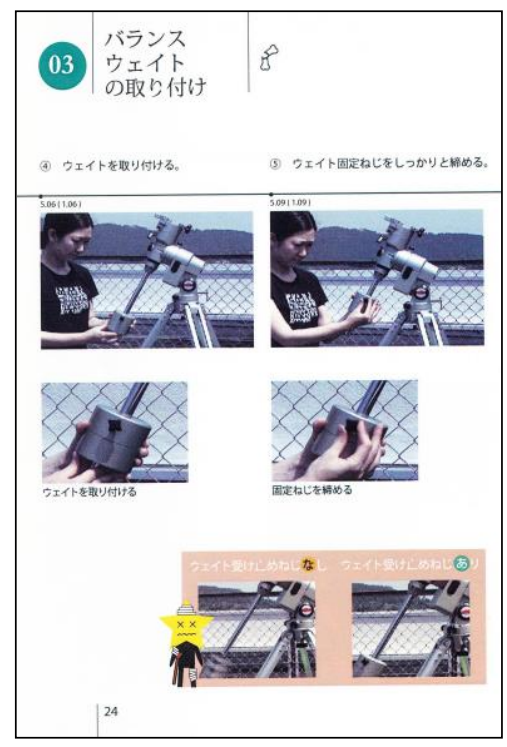

Figure 6. A page of the manual.

\section{Utilization of the teaching materials}

I have used my developed teaching materials at classes for students and training sessions of in-service teachers. The effects of the teaching materials are as follows:

(1) Improvement of the consciousness of the safety.

(2) Decrease of the frequency of the wrong acts.

\section{References}

1. Ministry of Education, Culture, Sports, Science and Technology, The course of study for elementary schools, p63-71, (Tokyo Shoseki, 2008)

2. Ministry of Education, Culture, Sports, Science and Technology, The course of study for elementary schools (English Ver.), http://www.mext.go.jp/a_menu/shotou/newcs/youryou/eiyaku/1261037.htm (2008)

3. Ministry of Education, Culture, Sports, Science and Technology, The course of study for junior high schools, p65-73, (Higashiyama Shobo, 2008)

4. Ministry of Education, Culture, Sports, Science and Technology, The course of study for junior high schools (English Ver.), http://www.mext.go.jp/a_menu/shotou/newcs/youryou/eiyaku/1298356.htm (2008) 